\title{
Retrospective study to evaluate the results of medical thoracoscopy in diagnosis of exudative pleural effusion at chest department El-Hussein university hospital in the period from July 2013 to July 2018
}

\author{
Ibrahim Abd El-Fattah Mohsen, Salim Mohammed Mohammed Abo-Sabe, Mahmoud Al- \\ Saeed Ahmed, Ahmed Adel Ibrahim Eaid
}

Department of Chest Diseases, Faculty of Medicine, Al-Azhar University (Cairo), Egypt

Corresponding author: Ahmed Adel Ibrahim Eaid, Email: ahmed.rona2@yahoo.com

\begin{abstract}
Background: Thoracoscopy is a minimally invasive procedure that allows visualization of the pleural space and intrathoracic structures. It enables taking pleural biopsies under direct vision, therapeutic drainage of effusions, and pleurodesis in one sitting. Persistent and recurrent exudative pleural effusions become common and thoracocentesis and blind pleural biopsy procedures do not give a definitive diagnosis in many patients. Therefore, thoracoscopy today remains the gold standard for these cases. In tuberculous pleuritis, the combined yield of histology and culture for rigid thoracoscopy was nearly $100 \%$.

Objective: This study was carried out to analyze our five years experience of medical thoracoscopy in the management of undiagnosed exudative pleural effusion at chest department El-Hussein University Hospital.

Materials and Methods: All patients with undiagnosed exudative pleural effusion who underwent thoracoscopy during the period between July 2013 to July 2018 at Al Hussein University Hospital were included in the study. All patients were subjected to thorough history taking, clinical examination, routine laboratory investigations, pleural fluid analysis and medical thoracoscopy with multiple pleural biopsies. All patients data, thoracoscopy results and complications were recorded.

Results: Thoracoscopy was successful for giving final diagnosis in 122 patients $(84.13 \%)$ from total 145 patients. Malignancies reported in 113 patients (77.9\%) of patients and TB reported in $6.2 \%$ of patients. Mesothelioma was the most common diagnosis in 67 patients(46.2\%) Minor complications occurred only in 14 out of 145 patients $(9.65 \%), 4$ patients $(2.8 \%)$ developed cellulitis, 8 patients $(5.5 \%)$ developed surgical emphysema, and 2 patient (1.4\%) developed bleeding.

Conclusion: Medical thoracoscopy is an easy, safe procedure with high diagnostic sensitivity for pleural effusion of uncertain etiology.
\end{abstract}

Keywords: Exudative pleural effusion, medical thoracoscopy, tuberculosis.

\section{INTRODUCTION}

Pleural effusion, pneumothorax, and pleural thickening are frequently encountered in pulmonary practice. Although the radiographic detection of pleural abnormalities may be obvious, determination of a specific diagnosis can present a challenge. Computed axial tomographic (CT) and ultrasound scans of the thorax and bronchoscopy with transbronchial biopsies may be helpful in selected circumstances, but the diagnostic yield for pleural disease is disappointingly low ${ }^{(1,2)}$. Percutaneous access to the pleural space is diagnostically useful and relatively simple; however, approximately 25 percent of pleural abnormalities remain undiagnosed after thoracentesis and/or closed pleural biopsies ${ }^{(3-6)}$. CTguided Abrams needle biopsy is a reasonable initial diagnostic procedure if pleural thickening is the main abnormality ${ }^{(7)}$. Thoracoscopy (or pleuroscopy) involves passage of an endoscope through the chest wall and offers the clinician a "window" for direct visualization and collection of samples from the pleura. It is a valuable diagnostic procedure and, in some cases, can also provide an opportunity for treatment ${ }^{(8)}$.
The aim of this study was to analyze our five years experience of medical thoracoscopy in the management of undiagnosed exudative pleural effusion at chest department El-Hussein University Hospital.

\section{MATERIALS AND METHODS}

This study was started in July 2013 and terminated at July 2018 at chest department, Al Hussein University Hospital. All patients who underwent thoracoscopy during this period were included in the study. Thoracoscopy was performed for diagnosis of exudative pleural effusions, which could not be detected by pleural fluid analyses using the routine biochemical, cytological, microbiological investigations and closed pleural biopsy. All patients underwent detailed clinical evaluation with history, clinical examination and radiological examination (CXR, CT). All the patients underwent the routine investigations including complete blood count and renal function tests. Prothrombin time, international normalized ratio, and platelet counts were done to rule out any bleeding tendency. 
Written informed consent was obtained from all the patients. The study was approved by the Ethics Board of Al-Azhar University.

\section{Thoracoscopy procedure:}

Patients were kept fasting for six hours prior to the procedure. Lateral decubitus position, with the side of pleural effusion facing upwards, was used for the procedure. Continuous monitoring of blood pressure and pulse rate was ensured during the procedure. Thoracoscopy was conducted under conscious sedation using intravenous aliquots of Midazolam along with local anesthesia (2\% solution of lidocaine). Skin was cleaned with antiseptic solution and draped with a sterilized cloth. In most of the cases, mid-axillary line was used for entry of thoracoscope, but in some patients, anterior or posterior axillary line were chosen based on clinical examination and radiological findings of loculation of fluid. A 1-2 $\mathrm{cm}$ skin incision was made, followed by a blunt dissection of intercostal muscles. A trocar with inner diameter of $8 \mathrm{~mm}$ was inserted through the chest wall followed by the insertion of the cannula through which the thoracoscope was introduced into the pleural cavity. Rigid thoracoscope by Karl Storz (model No. 1571721) was used. Pleural fluid was sucked out with suction catheter introduced through the working channel of thoracoscope. After achieving clear visualization, the pleural cavity was thoroughly inspected starting from diaphragmatic pleura as the first guide to the position of scope (because diaphragm movement helps with its easy identification). Adhesions, if present, were gently broken mechanically by moving thoracoscope or biopsy forceps through them. Any abnormal looking area was biopsied with the help of forceps introduced via working channel. Following the procedure, thoracoscope and cannula were removed and under water sealed drain was inserted. The demographic details like age, sex, smoking status, site of effusion, visual appearances on thoracoscopy, pathological diagnostic details were recorded. It was a crosssectional study.

\section{RESULTS}

This study included 145 patients with ranging age between 16-83 years and mean of $58.11 \pm 12.43$. Males were 81 patients $(55.9 \%)$ and females were 64 patients $(44.1 \%)$.
Table (1): Age distribution of the investigated sample across the study years (July 2013- July 2018).

\begin{tabular}{|c|c|c|c|c|c|c|}
\hline Variable & $\begin{array}{c}\text { 1st Year } \\
\mathbf{N o}=\mathbf{3 2}\end{array}$ & $\begin{array}{c}\text { 2nd Year } \\
\mathbf{N o}=\mathbf{3 3}\end{array}$ & $\begin{array}{c}\text { 3rd Year } \\
\mathbf{N o}=\mathbf{2 5}\end{array}$ & $\begin{array}{c}\text { 4th Year } \\
\mathbf{N o}=\mathbf{2 8}\end{array}$ & $\begin{array}{c}\mathbf{5} \text { th Year } \\
\mathbf{N o}=\mathbf{2 7}\end{array}$ & $\begin{array}{c}\text { Total } \\
\mathbf{N o}=\mathbf{1 4 5}\end{array}$ \\
\hline Age (years) & & & & & & \\
\hline Mean \pm SD & $54.69 \pm 13.06$ & $60.82 \pm 6.67$ & $59.12 \pm 10.89$ & $53.53 \pm 15.12$ & $63.96 \pm 10.99$ & $58.11 \pm 12.43$ \\
\hline Min-Max & $24-83$ & $47-72$ & $30-77$ & $16-75$ & $40-83$ & $16-83$ \\
\hline Statistics & \multicolumn{3}{|c|}{$\mathrm{F}=3.98$} & \multicolumn{3}{c|}{ P-value $=0.004 *$} \\
\hline
\end{tabular}

F: One-Way Analysis of Variance test. *: Significant.

Table (2): Gender distribution of the examined sample across the study years (July 2013- July 2018).

\begin{tabular}{|l|c|c|c|c|c|c|}
\hline \multicolumn{1}{|c|}{ Variable } & $\begin{array}{c}\text { 1st Year } \\
\text { No=32 (\%) })\end{array}$ & $\begin{array}{c}\text { 2nd Year } \\
\mathbf{N o = 3 3} \\
(\%)\end{array}$ & $\begin{array}{c}\text { 3rd Year } \\
\text { No=25(\%) }\end{array}$ & $\begin{array}{c}\text { 4th Year } \\
\text { No=28 (\%) }\end{array}$ & $\begin{array}{c}\text { 5th Year } \\
\mathbf{N o = 2 7}(\%)\end{array}$ & $\begin{array}{c}\text { Total } \\
\text { No=145 } \\
(\%)\end{array}$ \\
\hline Gender & & & & & & \\
\hline Male & $16(50.0)$ & $16(48.5)$ & $12(48.0)$ & $18(64.3)$ & $19(70.4)$ & $81(55.9)$ \\
\hline Female & $16(50.0)$ & $17(51.5)$ & $13(52.0)$ & $10(35.7)$ & $8(29.6)$ & $64(44.1)$ \\
\hline Statistics & \multicolumn{3}{|c|}{ X2=4.91 } & \multicolumn{3}{c|}{ P-value $=0.296$} \\
\hline
\end{tabular}

$\mathrm{X} 2$ : Chi-square test.

Table (3): Residence distribution of the explored sample across the study years (July 2013- July 2018).

\begin{tabular}{|l|c|c|c|c|c|c|}
\hline \multicolumn{1}{|c|}{ Variable } & $\begin{array}{c}\text { 1st Year } \\
\text { No=32 (\%) }\end{array}$ & $\begin{array}{c}\text { 2nd Year } \\
\text { No=33(\%) }\end{array}$ & $\begin{array}{c}\text { 3rd Year } \\
\text { No=25(\%) }\end{array}$ & $\begin{array}{c}\text { 4th Year } \\
\text { No=28(\%) }\end{array}$ & $\begin{array}{c}\text { 5th Year } \\
\text { No=27 (\%) }\end{array}$ & $\begin{array}{c}\text { Total } \\
\text { No=145 } \\
(\%)\end{array}$ \\
\hline Residence & & & & & & \\
\hline Shoubra El- & $12(37.5)$ & $13(39.4)$ & $8(32.0)$ & $8(28.6)$ & $10(37.0)$ & $51(35.2)$ \\
Khima & $8(25.0)$ & $5(15.1)$ & $2(8.0)$ & $3(10.7)$ & $3(11.1)$ & $21(14.5)$ \\
Helwan & $12(37.5)$ & $15(45.5)$ & $15(60.0)$ & $17(60.7)$ & $14(51.9)$ & $73(50.3)$ \\
Others & \multicolumn{3}{|c|}{$X 2=6.61$} & \multicolumn{3}{c|}{ P-value=0.580 } \\
\hline Statistics & \multicolumn{3}{|c|}{} \\
\hline
\end{tabular}

$\mathrm{X} 2$ : Chi-square test.

Table (4): Special habit distribution of the studied sample across the study years (July 2013- July 2018).

\begin{tabular}{|l|c|c|c|c|c|c|}
\hline Variable & $\begin{array}{c}\text { 1st Year } \\
\text { No=32(\%) }\end{array}$ & $\begin{array}{c}\text { 2nd Year } \\
\text { No=33(\%) }\end{array}$ & $\begin{array}{c}\text { 3rd Year } \\
\text { No=25(\%) }\end{array}$ & $\begin{array}{c}\text { 4th Year } \\
\text { No=28(\%) }\end{array}$ & $\begin{array}{c}\text { 5th Year } \\
\text { No=27 (\%) })\end{array}$ & $\begin{array}{c}\text { Total } \\
\text { No=145 } \\
(\%)\end{array}$ \\
\hline $\begin{array}{l}\text { Special } \\
\text { habit }\end{array}$ & & & & & & \\
\hline Smoker & $19(59.4)$ & $19(57.6)$ & $12(48.0)$ & $18(64.3)$ & $20(74.1)$ & $88(60.7)$ \\
\hline $\begin{array}{l}\text { Non- } \\
\text { smoker }\end{array}$ & $13(40.6)$ & $14(42.4)$ & $13(52.0)$ & $10(35.7)$ & $7(25.9)$ & $57(39.3)$ \\
\hline Statistics & \multicolumn{5}{|c|}{$\mathrm{X} 2=4.02$} & \multicolumn{3}{c|}{ P-value $=0.403$} \\
\hline
\end{tabular}

X2: Chi-square test. *: Significant.

Table (5): Gender distribution of smoking among the inspected sample across the study years (July 2013July 2018).

\begin{tabular}{|l|c|c|c|c|c|c|}
\hline Variable & $\begin{array}{c}\text { 1st Year } \\
\text { No=19(\%) }\end{array}$ & $\begin{array}{c}\text { 2nd Year } \\
\text { No=19(\%) }\end{array}$ & $\begin{array}{c}\text { 3rd Year } \\
\text { No=12 }(\%)\end{array}$ & $\begin{array}{c}\text { 4th Year } \\
\text { No=18 }(\%)\end{array}$ & $\begin{array}{c}\text { 5th Year } \\
\text { No=20 }(\%)\end{array}$ & $\begin{array}{c}\text { Total } \\
\text { No=88 } \\
(\%)\end{array}$ \\
\hline Smokers & & & & & & \\
\hline Male & $16(84.2)$ & $16(84.2)$ & $12(100.0)$ & $18(100)$ & $19(95.0)$ & $81(92.0)$ \\
\hline Female & $3(15.8)$ & $3(15.8)$ & $0(0.0)$ & $0(0.0)$ & $1(5.0)$ & $7(8.0)$ \\
\hline Statistics & \multicolumn{3}{|c|}{$\mathrm{X} 2=6.02$} & \multicolumn{3}{c|}{ P-value $=0.198$} \\
\hline
\end{tabular}

X2: Chi-square test. 
Table (6): Main complaint of the studied sample across the study years (July 2013- July 2018).

\begin{tabular}{|c|c|c|c|c|c|c|}
\hline $\begin{array}{c}\text { Main } \\
\text { complaint }\end{array}$ & $\begin{array}{c}\text { 1st Year } \\
\text { No=32 }(\%)\end{array}$ & $\begin{array}{c}\text { 2nd Year } \\
\text { No=33(\%) }\end{array}$ & $\begin{array}{c}\text { 3rd Year } \\
\text { No=25 (\%) }\end{array}$ & $\begin{array}{c}\text { 4th Year } \\
\text { No=28 }(\%)\end{array}$ & $\begin{array}{c}\text { 5th Year } \\
\text { No=27 }(\%)\end{array}$ & $\begin{array}{c}\text { Total } \\
\text { No=145 } \\
(\%)\end{array}$ \\
\hline Dyspnea & $25(78.1)$ & $28(84.8)$ & $20(80.0)$ & $24(85.7)$ & $19(70.4)$ & $116(80.0)$ \\
\hline Chest pain & $7(21.9)$ & $5(15.2)$ & $5(20.0)$ & $4(14.3)$ & $8(29.6)$ & $29(20.0)$ \\
\hline Statistics & \multicolumn{3}{|c|}{$\mathrm{X} 2=2.69$} & \multicolumn{3}{|c|}{ P-value $=0.611$} \\
\hline
\end{tabular}

$\mathrm{X} 2$ : Chi-square test.

This table shows the distribution of the main complaints in the studied group. The most common complaint was shortness of breath in 116 patients (80\%) while chest pain was the main complaint of 29 patients (20\%).

Table (7): Effusion amount of the scanned sample across the study years (July 2013- July 2018).

\begin{tabular}{|l|c|c|c|c|c|c|}
\hline $\begin{array}{c}\text { Effusion } \\
\text { amount }\end{array}$ & $\begin{array}{c}\text { 1st Year } \\
\text { No=32(\%) }\end{array}$ & $\begin{array}{c}\text { 2nd Year } \\
\text { No=33(\%) }\end{array}$ & $\begin{array}{c}\text { 3rd Year } \\
\text { No=25(\%) }\end{array}$ & $\begin{array}{c}\text { 4th Year } \\
\mathbf{N o = 2 8}(\%)\end{array}$ & $\begin{array}{c}\text { 5th Year } \\
\mathbf{N o = 2 7}(\%)\end{array}$ & $\begin{array}{c}\text { Total } \\
\text { No=145 } \\
(\%)\end{array}$ \\
\hline Massive & $9(28.1)$ & $10(30.3)$ & $11(44.0)$ & $6(21.4)$ & $7(25.9)$ & $43(29.7)$ \\
\hline Moderate & $23(71.9)$ & $23(69.7)$ & $14(56.0)$ & $22(78.6)$ & $20(74.1)$ & $102(70.3)$ \\
\hline Statistics & \multicolumn{3}{|c|}{$\mathrm{X} 2=3.60$} & \multicolumn{3}{c|}{ P-value $=0.463$} \\
\hline
\end{tabular}

$\mathrm{X} 2$ : Chi-square test.

This table shows the distribution of the effusion amount in the studied groups. There were 43 patients $(29.7 \%)$ with massive pleural effusion and $102(70.3 \%)$ with moderate pleural effusion.

Table (8): Effusion site of the examined sample across the study years (July 2013- July 2018).

\begin{tabular}{|l|c|c|c|c|c|c|}
\hline Effusion site & $\begin{array}{c}\text { 1st Year } \\
\text { No=32(\%) }\end{array}$ & $\begin{array}{c}\text { 2nd Year } \\
\text { No=33 (\%) }\end{array}$ & $\begin{array}{c}\text { 3rd Year } \\
\text { No=25 (\%) }\end{array}$ & $\begin{array}{c}\text { 4th Year } \\
\text { No=28(\%) }\end{array}$ & $\begin{array}{c}\text { 5th Year } \\
\text { No=27 (\%) })\end{array}$ & $\begin{array}{c}\text { Total } \\
\text { No=145 } \\
(\%)\end{array}$ \\
\hline Rt & $14(43.8)$ & $15(45.5)$ & $14(56.0)$ & $14(50.0)$ & $18(66.7)$ & $75(51.7)$ \\
\hline Lt & $17(53.1)$ & $17(51.5)$ & $11(44.0)$ & $14(50.0)$ & $9(33.7)$ & $68(46.9)$ \\
\hline Bilateral & $1(3.1)$ & $1(3.0)$ & $0(0.0)$ & $0(0.0)$ & $0(0.0)$ & $2(1.4)$ \\
\hline Statistics & \multicolumn{3}{|c|}{ X2=5.95 } & \multicolumn{5}{c|}{ P-value $=0.653$} \\
\hline
\end{tabular}

$\mathrm{X} 2$ : Chi-square test.

This table clarifies that the diseased side of the patients in the studied groups. There were 75 patients $(51.7 \%)$ right sided, 68 patients $(46.9 \%)$ left sided and bilateral in 2 patients $(1.4 \%)$.

Table (9): CT finding of the evaluated sample across the study years (July 2013- July 2018).

\begin{tabular}{|l|c|c|c|c|c|c|}
\hline \multicolumn{1}{|c|}{ CT finding } & $\begin{array}{c}\text { 1st Year } \\
\text { No=32 (\%) }\end{array}$ & $\begin{array}{c}\text { 2nd Year } \\
\mathbf{N o}=\mathbf{3 3}(\%)\end{array}$ & $\begin{array}{c}\text { 3rd Year } \\
\text { No=25 (\%) }\end{array}$ & $\begin{array}{c}\text { 4th Year } \\
\text { No=28 (\%) }\end{array}$ & $\begin{array}{c}\text { 5th Year } \\
\text { No=27(\%) }\end{array}$ & $\begin{array}{c}\text { Total } \\
\text { No=145(\%) }\end{array}$ \\
\hline Effusion only & $28(87.5)$ & $29(87.9)$ & $21(84.0)$ & $26(92.9)$ & $24(88.9)$ & $128(88.3)$ \\
\hline $\begin{array}{l}\text { Effusion+ } \\
\text { nodules }\end{array}$ & $3(9.4)$ & $2(6.1)$ & $3(12.0)$ & $0(0.0)$ & $2(7.4)$ & $10(6.9)$ \\
\hline $\begin{array}{l}\text { Effusion+ } \\
\text { adhesion }\end{array}$ & $1(3.1)$ & $2(6.1)$ & $1(4.0)$ & $2(7.1)$ & $0(0.0)$ & $6(4.1)$ \\
\hline $\begin{array}{l}\text { Effusion+ } \\
\text { thickening }\end{array}$ & $0(0.0)$ & $0(0.0)$ & $0(0.0)$ & $0(0.0)$ & $1(3.7)$ & $1(0.7)$ \\
\hline Statistics & \multicolumn{3}{|c|}{$\mathrm{X} 2=9.8$} & \multicolumn{5}{c|}{ P-value=0.633 } \\
\hline
\end{tabular}

$\mathrm{X} 2$ : Chi-square test.
This table demonstrates the CT finding, 128 patients $(88.3 \%)$ had pleural effusion only, 10 patients $(6.9 \%)$ had pl. effusion + nodules, 6 patients $(4.1 \%)$ had pl. effusion + adhesions and 1 patient $(0.7 \%)$ had pl. effusion + pl. thickening.

Table (10): Thoracoscopic findings of the studied sample across the tested years (July 2013- July 2018).

\begin{tabular}{|c|c|c|c|c|c|c|}
\hline $\begin{array}{l}\text { Thoracoscopic } \\
\text { finding }\end{array}$ & $\begin{array}{c}\text { 1st Year } \\
\text { No }=32(\%)\end{array}$ & $\begin{array}{c}\text { 2nd Year } \\
\text { No=33(\%) }\end{array}$ & $\begin{array}{c}\text { 3rd Year } \\
\text { No=25 }(\%)\end{array}$ & $\begin{array}{c}\text { 4th Year } \\
\text { No=28 }(\%)\end{array}$ & $\begin{array}{c}\text { 5th Year } \\
\text { No=27 }(\%)\end{array}$ & $\begin{array}{c}\text { Total } \\
\text { No=145 } \\
(\%)\end{array}$ \\
\hline Multiple nodules & $20(63.0)$ & $21(64.0)$ & $23(92.0)$ & $20(71.4)$ & $20(74.0)$ & $104(71.7)$ \\
\hline Pleural adhesions & $5(16.0)$ & $1(3.0)$ & $2(8.0)$ & $6(21.4)$ & $0(0.0)$ & $14(9.7)$ \\
\hline $\begin{array}{l}\text { Whitish costal } \\
\text { pleura }\end{array}$ & $1(3.0)$ & $0(0.0)$ & $0(0.0)$ & $0(0.0)$ & $0(0.0)$ & $1(0.7)$ \\
\hline $\begin{array}{l}\text { Pleural adhesions } \\
\text { and nodules }\end{array}$ & $5(16.0)$ & $6(18.0)$ & $0(0.0)$ & $2(7.1)$ & $0(0.0)$ & $13(9.0)$ \\
\hline $\begin{array}{l}\text { Pleural } \\
\text { thickening }\end{array}$ & $0(0.0)$ & $1(3.0)$ & $0(0.0)$ & $0(0.0)$ & $7(26.0)$ & $8(5.5)$ \\
\hline $\begin{array}{l}\text { Pleural adhesion } \\
\text { and plaque }\end{array}$ & $0(0.0)$ & $1(3.0)$ & $0(0.0)$ & $0(0.0)$ & $0(0.0)$ & $1(0.7)$ \\
\hline No abnormality & $1(3.0)$ & $3(9.0)$ & $0(0.0)$ & $0(0.0)$ & $0(0.0)$ & $4(2.8)$ \\
\hline Statistics & \multicolumn{3}{|c|}{$X 2=60.37$} & \multicolumn{3}{|c|}{$\mathrm{P}$-value $<0.001 *$} \\
\hline
\end{tabular}

$\mathrm{X} 2$ : Chi-square test. *: Significant.

This table reveals the thoracoscopic findings, 104 patients $(71.7 \%)$ had multiple nodules, 14 patients $(9.7 \%)$ had pleural adhesions, 1 patients $(0.7 \%)$ had Whitish costal pleura, 13 patients (9\%) had pl. adhesions + nodules, 8 patients $(5.5 \%)$ had pl. thickening, 1 patients $(0.7 \%) \mathrm{Pl}$. adhesions \& plaque, and 4 patients (2.8\%) No abnormality detected.

Table (11): Diagnosis of the studied sample across the surveyed years (July 2013- July 2018).

\begin{tabular}{|l|c|c|c|c|c|c|c|}
\hline \multicolumn{1}{|c|}{ Diagnosis } & $\begin{array}{c}\mathbf{1 s t} \text { Year } \\
\mathbf{N o = 3 2} \\
(\boldsymbol{\%})\end{array}$ & $\begin{array}{c}\mathbf{2 n d} \text { Year } \\
\mathbf{N o = 3 3} \\
(\boldsymbol{\%})\end{array}$ & $\begin{array}{c}\mathbf{3 r d} \text { Year } \\
\mathbf{N o = 2 5} \\
(\boldsymbol{\%})\end{array}$ & $\begin{array}{c}\text { 4th Year } \\
\mathbf{N o = 2 8} \\
(\%)\end{array}$ & $\begin{array}{c}\mathbf{5 t h} \text { Year } \\
\mathbf{N o = 2 7} \\
(\%)\end{array}$ & $\begin{array}{c}\text { Total } \\
\mathbf{N o = 1 4 5} \\
(\%)\end{array}$ & P-value \\
\hline Mesothelioma & $12(37.5)$ & $18(54.5)$ & $10(40.0)$ & $13(46.4)$ & $14(51.9)$ & $67(46.2)$ & 0.621 \\
\hline $\begin{array}{l}\text { Bronchogenic } \\
\text { Ca }\end{array}$ & $7(21.9)$ & $8(24.2)$ & $8(32.0)$ & $8(28.6)$ & $8(29.6)$ & $39(26.9)$ & 0.909 \\
\hline Adenocarcinoma & $6(18.8)$ & $7(21.2)$ & $4(16.0)$ & $7(25.0)$ & $7(25.9)$ & $31(21.4)$ & 0.892 \\
\hline $\begin{array}{l}\text { Squamous cell } \\
\text { Ca }\end{array}$ & $1(3.1)$ & $1(3.0)$ & $1(4.0)$ & $1(3.6)$ & $1(3.7)$ & $5(3.4)$ & 1.000 \\
\hline Small cell Ca & $0(0.0)$ & $0(0.0)$ & $1(4.0)$ & $0(0.0)$ & $0(0.0)$ & $1(0.7)$ & 0.305 \\
\hline $\begin{array}{l}\text { Bronchoalveolar } \\
\text { Ca }\end{array}$ & $0(0.0)$ & $0(0.0)$ & $2(8.0)$ & $0(0.0)$ & $0(0.0)$ & $2(1.4)$ & $0.045 *$ \\
\hline Lymphoma & $4(12.5)$ & $0(0.0)$ & $1(4.0)$ & $0(0.0)$ & $1(3.7)$ & $6(4.1)$ & 0.082 \\
\hline Thymoma & $1(3.1)$ & $0(0.0)$ & $0(0.0)$ & $0(0.0)$ & $0(0.0)$ & $1(0.7)$ & 0.469 \\
\hline TB & $2(6.3)$ & $3(9.1)$ & $1(4.0)$ & $2(7.1)$ & $1(3.7)$ & $9(6.2)$ & 0.908 \\
\hline Non-specific1 & $6(18.8)$ & $4(12.1)$ & $5(20.0)$ & $5(17.9)$ & $3(11.1)$ & $23(15.9)$ & 0.843 \\
\hline
\end{tabular}

P-value by: Chi-square test. *: Significant.

This table displays the histopathological type of malignancy in the diagnosed patients by the thoracoscope in the studied sample. The most common diagnosis was malignant pleural mesothelioma in 67 patients $(46.2 \%)$ then metastatic adenocarcinoma in 31 patients $(21.4 \%)$ while5 
patients (3.4\%) had squamous cell carcinoma, 1 patients $(0.7 \%)$ had small cell carcinoma, 2 patients (1.4\%) had Bronchoalveolar carcinoma. 6 patients (4.1\%) had lymphoma, 1 patients $(0.7 \%)$ had Thymoma, 9 patients $(6,2 \%)$ had TB and 23 (15.9\%) nonspecific diagnosis.

Table (12): Thoracoscopic complications of the studied sample across the analyzed years (July 2013July 2018).

\begin{tabular}{|l|c|c|c|c|c|c|}
\hline Complications & $\begin{array}{c}\text { 1st Year } \\
\mathbf{N o = 3 2}(\%)\end{array}$ & $\begin{array}{c}\text { 2nd Year } \\
\mathbf{N o = 3 3}(\%)\end{array}$ & $\begin{array}{c}\text { 3rd Year } \\
\mathbf{N o = 2 5}(\%)\end{array}$ & $\begin{array}{c}\text { 4th Year } \\
\mathbf{N o}=\mathbf{2 8}(\%)\end{array}$ & $\begin{array}{c}\text { 5th Year } \\
\mathbf{N} \mathbf{2}=\mathbf{2 7}(\%)\end{array}$ & $\begin{array}{c}\text { Total } \\
\mathbf{N o = 1 4 5} \\
(\%)\end{array}$ \\
\hline Cellulitis & $3(9.4)$ & $0(0.0)$ & $0(0.0)$ & $0(0.0)$ & $1(3.7)$ & $4(2.8)$ \\
\hline S. emphysema & $1(3.1)$ & $1(3.0)$ & $1(4.0)$ & $3(10.7)$ & $2(7.4)$ & $8(5.5)$ \\
\hline Bleeding & $1(3.1)$ & $0(0.0)$ & $1(4.0)$ & $0(0.0)$ & $0(0.0)$ & $2(1.4)$ \\
\hline No & $27(84.4)$ & $32(97.0)$ & $23(92.0)$ & $25(89.3)$ & $24(88.9)$ & $131(90.3)$ \\
\hline Statistics & \multicolumn{3}{|c|}{ X2=13.36 } & \multicolumn{3}{c}{ P-value $=0.343$} \\
\hline
\end{tabular}

X2: Chi-square test.

This table shows that complications occurred only in 14 out of 145 patients $(9.65 \%), 4$ patients $(2.8 \%)$ were complicated by cellulitis, 8 patients (5.5\%) were complicated by surgical emphysema, 2 patients $(1.4 \%)$ were complicated by bleeding and 131 patients (90.3) had no post thoracoscopic complications.

\section{DISCUSSION}

In the absence of thoracoscopy, pleural fluid cytology and closed pleural biopsy are the two commonly applied procedures for the diagnosis of pleural effusion. The diagnostic yield of cytology in malignant pleural effusion varies between $30 \%$ and $80 \%{ }^{(9)}$. In pleural effusions undiagnosed by thoracocentesis, closed pleural biopsy increases the yield by about $10 \%$ and $40 \%$ in malignant and tuberculous pleural effusions, respectively. However, the diagnostic yield of thoracoscopy is about $93 \%$ in both malignant and tuberculous pleural effusions ${ }^{(10,11)}$.

Thoracoscopy is an excellent diagnostic procedure. The added advantage in thoracoscopy is the direct visualization of biopsied areas, which can increase the diagnostic yield ${ }^{(10)}$.

The present study included 145 patients with age ranging from $16-83$ years and mean of $58.11 \pm 12.43$. Males were 81 patients $(55.9 \%)$ and females were 64 patients $(44.1 \%)$.

This study showed that most of patients 51 patients (35.2\%) living at Shoubra El-Khima, 21 patients (14.5\%) living at Helwan and 73 patients $(50.3 \%)$ other patients from different sites.

In this study, 88 patients $(60.7 \%)$ were found smoker and 57 patients $(39.3 \%)$ were nonsmoker. 81 patients $(92 \%)$ of them were males while 7 patients were females.

The commonest compliant in our patients was dyspnea (80\%) and atypical chest pain $(20 \%)$.

Moreover, it was found that, 43 patients (29.7\%) had massive pleural effusion while, 102 patients $(70.3 \%)$ had moderate pleural effusion.

In this study, 75 patients $(51.7 \%)$ had right sided pleural effusion while, 68 patients $(46.9 \%)$ had left sided pleural effusion and 2 patients $(1.4 \%)$ had bilateral pleural effusion(diagnosed as mesothelioma).

As regard to computed tomographic CT findings, 128 patients $(88.3 \%)$ had pleural effusion only, 10 patients $(6.9 \%)$ had pl. effusion + nodules, 6 patients $(4.1 \%)$ had pl. effusion + adhesions and 1 patient had pl. effusion + pl. thickening $(0.7 \%)$.

Thoracoscopic findings, showed that 104 patients $(71.7 \%)$ had multiple nodules, 14 patients (9.7\%) had pleural adhesions, 13 patients $(9 \%)$ had pl. adhesions + nodules, 8 patients $(5.5 \%)$ had pl. thickening, 1 patients (.7\%) Pl. adhesions \& plaque, 1patients (.7\%) Whitish costal pleura and 4 patients $(2.8 \%)$ No abnormality detected.

The present work revealed that thoracoscopy was successful for giving final diagnosis in 122 patients $(84.13 \%)$ from total 145 patients. Our results are in agreement with those of Patil et al. ${ }^{(12)}$ through a study conducted at Departments of Pathology and Respiratory Medicine, J.L.N. Medical College, Ajmer, Rajasthan, India on 129 patients with undiagnosed exudative pleural effusions medical thoracoscopy. This study, gave a definitive diagnosis in 110 out of 129 patients with a diagnostic yield of $(85.3 \%)$, Hucker et al. ${ }^{(13)}$ from England reported a diagnostic sensitivity of $80.3 \%$ in their study which included 102 patients, Mootha et al. ${ }^{(14)}$ from India reported yield of $74.3 \%$ in 35 patients, Dhanya et al. ${ }^{(15)}$ from Thailand could achieve diagnosis with pleural biopsy in $95.8 \%$ of patients and Hansen et $\boldsymbol{a l}^{\left({ }^{(16)}\right.}$ from Denmark who were able to achieve diagnosis in $90.4 \%$ in a total of 147 patients of undiagnosed pleural effusion. 
In the present study malignancies were reported in $113(77.9 \%)$ of patients, nonspecific in 23 patients (15.9\%) and TB in 9 patients (6.2\%). This is in agreement with a study by Mohamed et al. ${ }^{(17)}$ who found that the most common diagnosis was malignancy in $87 / 117$ patients $(74.3 \%$ ), followed by chronic nonspecific pleurisy in 16/117 patients (13.6\%), tuberculous pleurisy in five patients (4.27\%), septic empyema in six patients (5.13\%), sarcoidosis in two patients (1.7\%), and systemic lupus erythematosus in one patient $(0.85 \%)$.

As regard to histopathological type of malignancy which were discovered in the present study, the most common diagnosis was malignant pleural mesothelioma in 67 patients $(46.2 \%)$, the second common diagnosis coming after mesothelioma. In the present study was malignant metastatic adenocarcinoma was observed in 31 patients (21.4\%), 5 patients (3.4\%) had squamous cell carcinoma, 1 patients $(0.7 \%)$ had small cell carcinoma, 2 patients $(1.4 \%)$ had Bronchoalveolar carcinoma. 6 patients $(4.1 \%)$ had lymphoma, 1 patients $(0.7 \%)$ had Thymoma.

The present conclusions seem to concur those found by Helala et al. ${ }^{(18)}$ who revealed that the most common pathological type was MPM in $15 / 28$ patients $(53.6 \%$ ), followed by metastatic adenocarcinoma in 10/28 patients (in 35.6\%).

In the current study, according to the residence of the studied patients, we found that the majority of the patients are living in Shoubra ElKheima and Helwan. Most of patients from these areas diagnosed with malignant mesothelioma. It means that environmental exposure to asbestos has a relationship with mesothelioma in patients working or living in the neighborhood with asbestos factories. The patients diagnosed as mesothelioma 38(74.5\%) patients from Shoubra ElKhima, 11(52.4\%) from Helwan and 18(24.7\%) from other places. The patients diagnosed as bronchogenic carcinoma 3(5.9\%) patients from Shoubra El-Khima, 6(28.6\%) from Helwan and $30(41.1 \%)$ from other places. No statistical significance differences were observed between Shoubra El-Khima, Helwan and other places regarding lymphoma, Thymoma, TB pleurisy and nonspecific. This remark is supported by the study conducted by Ibrahim et al. $^{(19)}$ and colleagues in Kasr Al Aini Center of Radiation Oncology and Nuclear Medicine, Kasr Al Aini School of Medicine, Cairo University. It included retrospective cases of malignant mesothelioma presenting to the Palliative Care Unit from January 2009 to December 2011. They were diagnosed in the period between June 2005 and July 2011, which revealed 15 cases $(37.5 \%)$ of mesothelioma from Shoubra and eight cases (20\%) from Helwan In their study.

In present study, complication occurred only in 14 out of 145 patients $(9.65 \%), 4$ patients $(2.8 \%)$ were complicated by cellulitis, 8 patients $(5.5 \%)$ were complicated by surgical emphysema, 2 patients $(1.4 \%)$ were complicated by bleeding and 131 patients (90.3) had no post thoracoscopic complications. This correlates with findings of Prabhu et al. (20) who reported no major complications out of 68 patients; only $4 / 68$ patients (5.8\%) had minor complications, like subcutaneous emphysema in $3 / 68$ patients $(4.4 \%$ ) and prolonged air leak in $1 / 68(1.4 \%)$ patient, This also correlates with the study of Ahmed et al. ${ }^{(21)}$ who reported that $4 \%$ of patients were complicated by cellulitis, $4 \%$ were complicated by surgical emphysema, $2 \%$ were complicated by bronchopleural fistula and $90 \%$ had no complication.

\section{CONCLUSION}

It was found that medical thoracoscopy is an easy procedure and an excellent diagnostic procedure for pleural effusion of uncertain etiology. It has low complication rate even in settings where the procedure is just started. It should be included in the armamentarium of procedures for management of pleural effusion.

\section{REFERENCES}

1. Pugatch R, Faling L, Robbins A, Snider G (1978): Differentiation of pleural and pulmonary lesions using computed tomography, J. Comput. Assist Tomogr., 2:601.

2. Feinsilver S, Barrows A, Braman S (1986): Fiberoptic bronchoscopy and pleural effusion of unknown origin, Chest, 90:516.

3. Poe R, Israel R, Utell M et al. (1984): Sensitivity, specificity, and predictive values of closed pleural biopsy, Arch Intern Med., 144:325.

4. Ryan C, Rodgers R, Unni K, Hepper $\mathbf{N}$ (1981): The outcome of patients with pleural effusion of indeterminate cause at thoracotomy, Mayo Clin Proc., 56:145. 
5. Boutin $\mathbf{C}$, Viallat $\mathbf{J}$, Cargnino $\mathbf{P}$, Farisse $\mathbf{P}$ (1981): Thoracoscopy in malignant pleural effusions, Am. Rev. Respir. Dis., 124:588.

6. Boutin C, Cargnino P, Viallat J (1980): Thoracoscopy in the early diagnosis of malignant pleural effusions, Endoscopy, 12:155.

7. Metintas M, Ak G, Dundar E et al. (2010): Medical thoracoscopyvs CT scan-guided Abrams pleural needle biopsy for diagnosis of patients with pleural effusions: a randomized, controlled trial, Chest, 137:1362.

8. Rodriguez-Panadero F, Janssen J, Astoul P (2006): Thoracoscopy: general overview and place in the diagnosis and management of pleural effusion, Eur Respir J., 28:409.

9. Emad A, Rezaian G (1998): Diagnostic value of closed percutaneous pleural biopsy vs pleuroscopy in suspected malignant pleural effusion or tuberculous pleurisy in a region with a high incidence of tuberculosis, a comparative, agedependent study. Respir. Med., 92:488-92.

10. Blanc F, Atassi K, Bignon J, Housset B (2002): Diagnostic value of medical thoracoscopy in pleural disease: a 6- year retrospective study, Chest, 121:1677-83.

11. Rahman N, Ali N, Brown G, Chapman S, Davies R, Downer $N$ et al. (2010): Local anaesthetic thoracoscopy: British Thoracic Society Pleural Disease Guideline 2010, Thorax, 65:ii54-60.

12. Patil C.B, Dixit R, Gupta R, Gupta N, Indushekar V (2016): Thoracoscopic evaluation of 129 cases having undiagnosed exudative pleural effusions, Lung India, 33:502-6.

13. Hucker J, Bhatnagar NK, al Jilaihawi AN, Forrester CP (1991): Thoracoscopy in the diagnosis and management of recurrent pleural effusion,. Ann. Thorac. Surg., 52:114-7.

14. Mootha V, Agarwal R, Singh N, Aggarwal A, Gupta D, Jindal S (2011): Medical thoracoscopy for undiagnosed pleural effusions: Experience from a tertiarycare hospital in north India, Indian J. Chest Dis. Allied Sci., 53:21-4.

15. Dhanya T, Ravindran C (2009): Medical Thoracoscopy - Minimally invasive diagnostic tool for a trained Pulmonologist, Calicut. Med. J., 7:e4.
16. Hansen M, Faurschoul, Clementsen $P$ (1998): Medical thoracoscopy: results and complications in 146 patients. A retrospective study. Respir Med., 92: 228-32.

17. Mohamed S, Shaban M (2014): Diagnostic yield of medical thoracoscopy in diagnosis of exudative pleural effusion, one year prospective study, J. Chest Dis. Tuberc., 63:897-905.

18. Helala L, El-Assal G, Farghally A, Abd El Rady M (2014): Diagnostic yield of medical thoracoscopy in cases of undiagnosed pleural effusion in Kobri El-Kobba Military Hospital, Egypt J. Chest Dis. Tuberc., 63:629-634.

19. Ibrahim N, Abou-Elela E, Darwish D (2013): Survival of mesothelioma in a palliative medical care unit in Egypt, Asian Pac. J. Cancer Prev., 14(2):739-742.

20. Prabhu V, Narasimhan R (2012): The role of pleuroscopy in undiagnosed exudative pleural effusion, Lung India, 29:128-130.

21. Ahmed M, Halim A, Aziz A, El-Shorpagy $R$ (2016): Outcomes and complications of medical thoracoscopy in undiagnosed exudative pleural effusion, J. Broncol., 10:93-99. 